\title{
A CONVERSION FORMULA TO CORRECT FOR PULSE OXIMETER DESATURATIONS OBTAINED WITH DIFFERENT AVERAGING TIMES
}

\author{
J. Vagedes ${ }^{1}$, T.W. Vine ${ }^{2}$, C. Wiechers ${ }^{1}$, F.A. Hanel ${ }^{1}$, C.F. Poets ${ }^{1}$, K. Dietz ${ }^{3}$
}

${ }^{I}$ Children's Hospital, Department of Neonatology, University of Tübingen, Tübingen, ${ }^{2}$ ARCIM-Institute, Research Department, Filderklinik, Filderstadt, ${ }^{3}$ Department of Medical Biometry, University of Tübingen, Tübingen, Germany

Study objectives: Pulse oximetry has gained widespread use in neonatology and intensive care medicine to measure arterial oxygen saturation $\left(\mathrm{SpO}_{2}\right)$ continuously. The $\mathrm{SpO} 2$ values are usually obtained by averaging over preceding measurements. As the averaging time, usually between 2 to 16 seconds, affects the number of desaturations, it is necessary to have a conversion formula to compare results obtained using different averaging times.

Methods: Oxygen saturation was recorded for 168 hours in fifteen infants with a mean number 60.9 desaturations $<90 \%$ per hour for using a pulse oximeter (Radical, Masimo). The raw data were reprocessed using 7 different averaging times between 2 and 16 seconds to determine the number of desaturations (D) below the threshold values of $80 \%, 85 \%$ or $90 \%$ for 7 different minimal desaturation durations $(>0,>5,>10$, $>15,>20,>25,>30$ seconds).

Measurements and results: We found a linear relationship between the logarithm of the desaturation rate and the logarithm of the averaging time. Based on this linear relationships the conversion formula is: $D_{2}=D_{1}$ * $\left(T_{2} / T_{1}\right)^{C}$, where $D_{2}$ is the desaturation rate for the desired averaging time $T_{2}$ and $D_{1}$ is the desaturation rate for the original averaging time $\mathrm{T}_{1}$, where the exponent $\mathrm{c}$ depends on the desaturation threshold and the minimal desaturation duration. The median percentage error was found to be $2.5 \%$.

Conclusion: A formula is presented to convert between the numbers of desaturations measured with different averaging times for different desaturation thresholds and minimal durations. 